Article

\title{
Ferroelectric Liquid Crystal Compound Lens Based on Pancharatnam-Berry Phase
}

\author{
Ying Ma ${ }^{1, *}$, Mingkui Yin ${ }^{1}$, Yuhang Shan ${ }^{1}$, Vladimir G. Chigrinov ${ }^{2}{ }^{(0}$, Hoi-Sing Kwok $^{3}$ and Jianlin Zhao ${ }^{1}(\mathbb{C}$ \\ 1 Key Laboratory of Light Field Manipulation and Information Acquisition, Ministry of Industry and \\ Information Technology, School of Physics Science and Technology, Northwestern Polytechnical University, \\ Xi'an 710129, China; mkyin0801@mail.nwpu.edu.cn (M.Y.); 2016302754@mail.nwpu.edu.cn (Y.S.); \\ jlzhao@nwpu.edu.cn (J.Z.) \\ 2 Nanjing Nanhui Intelligent Optical Sensing and Manipulation Research Institute Co., Ltd., \\ Nanjing 210093, China; eechigr@ust.hk \\ 3 State Key Laboratory (SKL) on Advanced Displays and Optoelectronics Technologies, \\ Department of Electronic and Computer Engineering, Hong Kong University of Science and Technology, \\ Kowloon 999077, Hong Kong; eekwok@ust.hk \\ * Correspondence: maying916@nwpu.edu.cn
}

check for updates

Citation: Ma, Y.; Yin, M.; Shan, Y.; Chigrinov, V.G.; Kwok, H.-S.; Zhao, J. Ferroelectric Liquid Crystal Compound Lens Based on Pancharatnam-Berry Phase. Crystals 2022, 12, 231. https://doi.org/ $10.3390 /$ cryst 12020231

Academic Editor: Ingo Dierking

Received: 23 December 2021

Accepted: 31 January 2022

Published: 8 February 2022

Publisher's Note: MDPI stays neutral with regard to jurisdictional claims in published maps and institutional affiliations.

Copyright: (C) 2022 by the authors. Licensee MDPI, Basel, Switzerland. This article is an open access article distributed under the terms and conditions of the Creative Commons Attribution (CC BY) license (https:// creativecommons.org/licenses/by/ $4.0 /)$.

\begin{abstract}
We report a ferroelectric liquid crystal (FLC) compound lens based on the PancharatnamBerry (PB) phase. The phase of the FLC compound lens is an integration of polarization grating and a PB lens. Thus, when light passes through an FLC compound lens, the output light's polarization handedness will be changed accordingly. In this case, FLC compound lenses can function as concave/convex lenses with spatially separated output light and rapid transmittance tunability. The FLC compound lenses were fabricated using a single-step holographic exposure system, based on a spatial light modulator working as numerous phase retarders. Photosensitive azo-dye material was used as the aligning layer. The output light transmittance of the FLC compound lens can be operated at $150 \mu$ s. Our results achieve the potential applications on various displays and augmented reality.
\end{abstract}

Keywords: ferroelectric liquid crystals; Pancharatnam-Berry phase; compound lens

\section{Introduction}

The lens, as one of the most important optical elements, is widely used in our daily life for optical usages, such as the eyeglass, camera objective, microscope and telescope [1]. Compared with conventional refractive lenses, liquid crystal (LC) lenses show good potential, with the features of controllable birefringence and refractive indices, as well as high integration and compact size. In recent years, Pancharatnam-Berry (PB) phase devices have been regarded as the fourth generation of optical elements in the optical science and engineering community, where the circular polarization-dependent devices are well utilized, including the capacity of optical communication systems [2,3], and virtual and augmented reality displays [4-7]. The PB phase devices fabricated with liquid crystals (LCs) are potential candidates because of their inherent photoelectric properties, large optical birefringence and controllable continuous orientation [8,9]. Several well-known optical elements use the PB phase, including the PB lens [10,11], polarization grating (PG) [12] and the q-plate [13]. They are widely used for the shaping of various wavefronts, such as a helical wavefront for vortex beams [9] and vector beams [14], as well as laser beam shaping [15]. With the help of LC photoalignment technology [16,17], it is becoming possible to realize complex micro-sized LC structures. Ferroelectric liquid crystals (FLCs) utilizing photo-alignment technology $[16,18-20]$ represent a promising way to produce LC PB devices with a microsecond response [21], which is at least two orders of magnitude higher than common nematic liquid crystals. Several mask-free photopatterning systems have been developed. A DMD (digital mirror device) [21-24] and direct laser writing [25-27] 
were reported, but failed to provide single-step exposure. Recently, a single-step exposure system was used to fabricate PB gratings and q-plates based on a spatial light modulator (SLM) [28]. A fast-switching PB lens fabricated with ferroelectric liquid crystals (FLCs), based on an SLM exposure system, was also realized [21].

For the general LC PB lens, bifocal property has been realized according to the different handedness of the incident circularly polarized light [29]. In this paper, we have demonstrated an FLC compound lens, and deformed helix FLCs (DHFLCs) were selected because they can offer a fast response, continuously changing light transmittance [30] and a defect-free structure. Different from the conventional FLC PB lens, the proposed FLC compound lens can switch between two spatially separated focuses, manipulating light by the generation and modulation of the PB phase [31,32], with the fast response property of FLCs. With these special optical properties, the lens shows good potential to be used in many modern applications, such as polarizing imaging $[33,34]$, micro particle manipulation [35] and multifocal cameras that can control the focus of the generated beams or the positions of the generated light paths [36]. The diffraction efficiency of FLC compound lenses can be rapidly changed with a switching-on time of $150 \mu$ s at $4 \mathrm{~V} / \mu \mathrm{m}$. The output light passing through an FLC lens shows precise, spatial separation with different focuses, which are in good agreement with the theoretical results. Light beam separation angles and focuses can be designed based on different applications.

\section{Materials and Methods}

\subsection{Materials}

With the help of the realization of the photoalignment method, a complex FLC aligning structure became possible. A polarization photosensitive alignment sulphonic azo-dye (SD1) was chosen as the aligning material for its flexible anchoring energy, lack of mechanical damage and minimized unwanted electronic charges. Under exposure from a polarized light source with a UV-to-blue spectrum, the SD1 molecule tends to orient perpendicularly to the polarization of the incident light. Its working principle and molecular structure are shown in Figure 1a,b. The blue cylindrical objects at the bottom represent liquid crystal molecules, whereas the yellow arrows above represent the polarization state of light, and the molecular orientations in different color regions can be different when polarizations of incident light are region dependent.

(a)

(c)
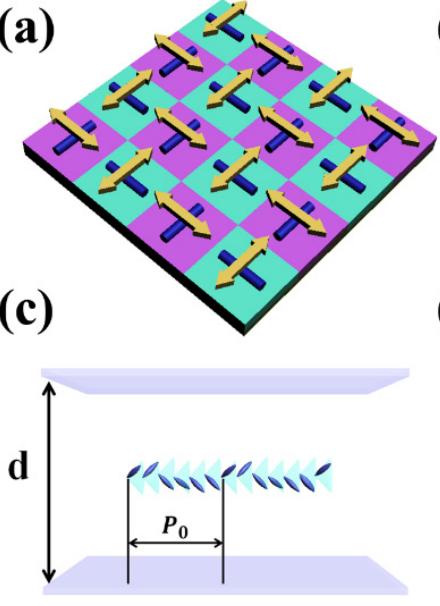

(b)

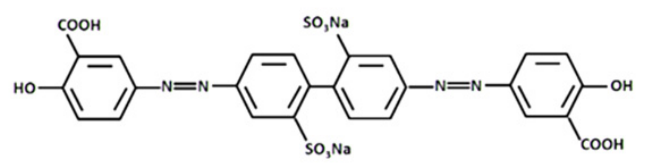

(d)

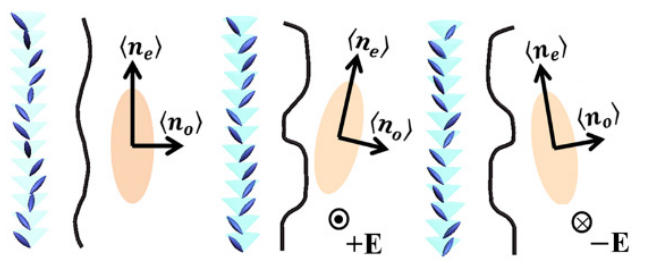

Figure 1. (a) SD1 with (b) its formula, providing the alignment direction perpendicular to the exposure polarization of the incident light. (c) Structure of a DHFLC cell and the rotating directions of the FLC molecules with external electric field. (d) Birefringence mechanism of DHFLC.

In this work, DHFLCs [37] were selected because they can offer a fast response and continuously changing light transmittance $[21,30,38]$. In particular, DHFLC 587 (from P.N. Lebedev Physical Institute of Russian Academy of Sciences, Moscow, Russia) was selected 
for its high transmission in the visible spectrum. The phase transition sequence of this LC when heated up from the solid crystalline phase is $\mathrm{Cr} \rightarrow 12{ }^{\circ} \mathrm{C} \rightarrow \mathrm{SmC}^{*} \rightarrow 110^{\circ} \mathrm{C} \rightarrow$ $\mathrm{SmA}^{*} \rightarrow 127^{\circ} \mathrm{C} \rightarrow$ Is, while cooling from smectic $\mathrm{C}^{*}$ phase crystallization occurs around $-10-15{ }^{\circ} \mathrm{C}$. The spontaneous polarization $P$ s and the tilt angle $\theta$ at room temperature are $150 \mathrm{nC} / \mathrm{cm}^{2}$ and $36.5^{\circ}$, respectively. $n_{e}$ and $n_{0}$ are 1.67 and 1.57 measured at room temperature. Two optically flat indium tin oxide-coated glass plates were used for preparing a sandwich-type sample holder. SD1 was coated by a spin coater at $3000 \mathrm{r} / \mathrm{min}$ for $30 \mathrm{~s}$. Spacers were used to form the sandwich-like structure. The designed pattern was exposed on the cell using an exposure system, which will be illustrated in the following section. FLC was then filled into the cell when the cell was heating up to its isotropic temperature. The cell thickness was $3 \mu \mathrm{m}$, calculated in order to satisfy the half wave condition using $\Delta n d=\lambda / 2$, where $\Delta \mathrm{n}$ represents the birefringence and $\lambda=633 \mathrm{~nm}$. In the schematic of a typical DHFLC cell shown in the upper picture of Figure 1c, when there is no electric field, a helix exists with helix pitch denoted as $P_{0}$ and one important requirement for DHF effect existence is $d \gg P_{0}$. When there is an external electric field to DHFLC molecules, the fast axis changes in accordance with the absolute value of the electric field. The principle of birefringence of a DHFLC is shown in Figure 1d. The long axis of the index ellipsoid is along the $z$-axis in the absence of an electric field. The refractive index ellipsoid rotates in the plane perpendicular to the electric field, and its long axis is offset relative to the $z$-axis when the electric field exists. When the polarity of the electric field is positive, the DHFLC molecules are aligned along the cone surface to the right, and the refractive index ellipsoid rotates to the right. The DHFLC molecules and the refractive index ellipsoid rotate to the other side when the polarity of the electric field is opposite. The birefringence is described by the following equation:

$$
\Delta n(E)=\sqrt{\left\langle\frac{n_{\|}^{2} n_{\perp}^{2}}{n_{\perp}^{2}+\left(n_{\|}^{2}-n_{\perp}^{2}\right) \sin ^{2} \theta \sin ^{2} \varphi(E)}\right\rangle}-n_{\perp}
$$

where $n_{\|}$and $n_{\perp}$ can be considered as $n_{e}$ and $n_{0}$ without the electric field. $n_{\|}, n_{\perp}$ and the azimuth angle $\varphi$ change in the presence of the electric field. Transmittance can then be calculated based on the parameters of the certain FLC material [39].

\subsection{Fabrication Method}

The exposure system based on SLM is depicted in Figure 2a, which consists of a $450 \mathrm{~nm}$ laser, two optical lenses (Lens1 and Lens2), a half-wave plate (HWP), a rightangle prism, an SLM and a quarter-wave plate (QWP). Its working principal is to use an SLM as several light beam retarders to generate regional, polarized output light, which is described in detail in our previous work [39]. The polarizations of output light from the SLM can be precisely controlled by the electric field loaded through computer-designed grayscale images. The final polarized output light can be exposed on the SD1-coated liquid crystal cells.

\subsection{Theory}

Both the PB lens and the liquid crystal PGs are PB phase liquid crystal devices. Their phases are described by $\varphi 1$ and $\varphi 2$, respectively, as follows:

$$
\begin{gathered}
\varphi 1=\frac{2 \pi}{\lambda}\left(\sqrt{x^{2}+y^{2}+f^{2}}-f\right) \\
\varphi 2=\frac{2 \pi x}{\Lambda}
\end{gathered}
$$

where $\lambda$ is the wavelength of the incident light, $f$ is the focus length of the lens, $\Lambda$ is the period of the polarization grating, and $x$ and $y$ are the coordinates of the wavefront. Through $\varphi 1$ and $\varphi 2$, we can observe that the lens can diverge or converge the light and the 
polarization grating can change the direction of the light. By superimposing $\varphi 1$ and $\varphi 2$, it is possible to realize the integration of the two functions. The phase of the integrated device is expressed as $\varphi$ in the following:

$$
\varphi=\frac{2 \pi}{\lambda}\left(\sqrt{x^{2}+y^{2}+f^{2}}-f\right)+\frac{2 \pi x}{\Lambda}
$$

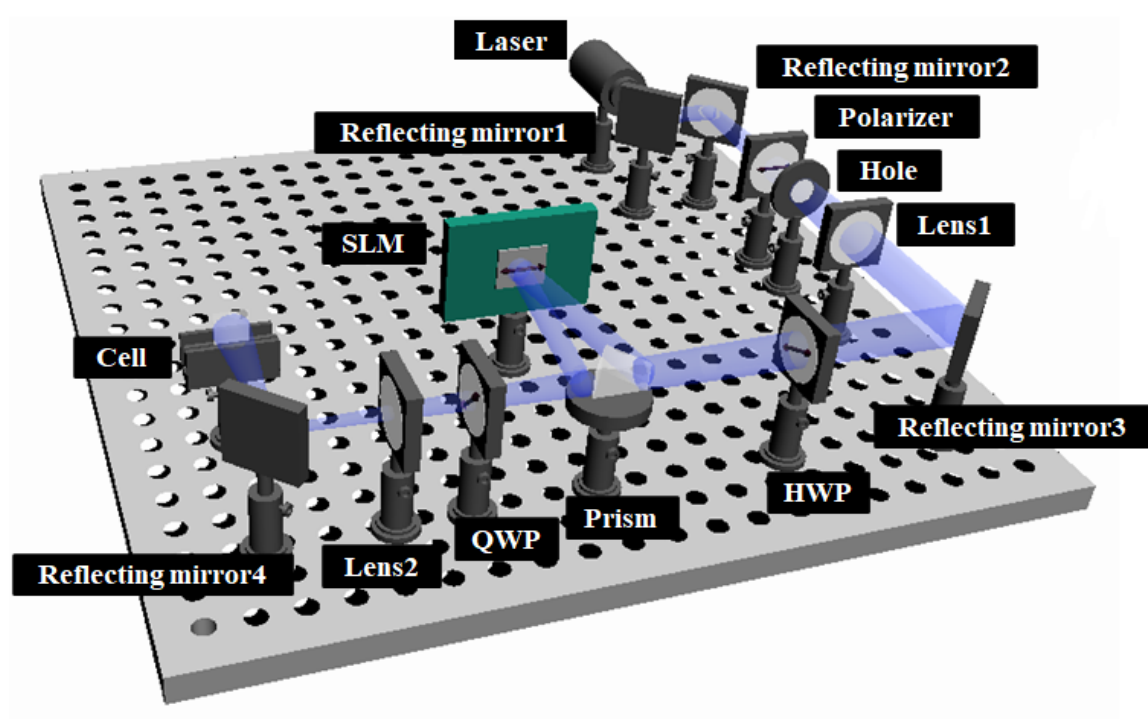

(a)

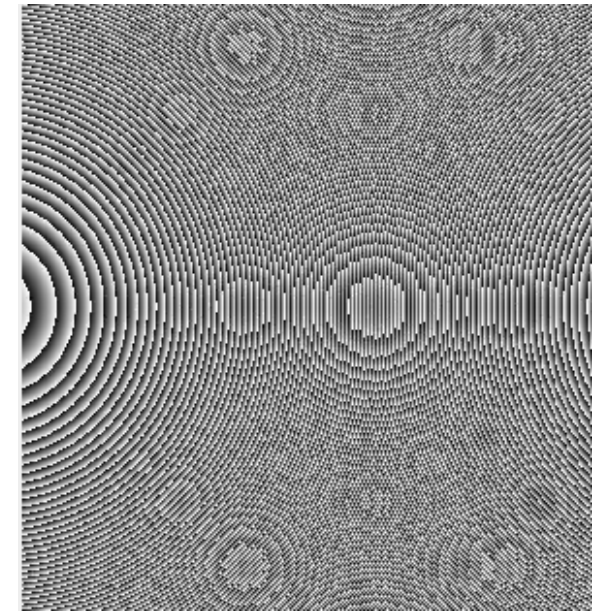

(b)

Figure 2. (a) Experimental setup of the single-step holographic exposure system used for a photosensitive azo-dye material SD1. (b) Simulation result of grayscale image loaded onto the SLM.

The integrated device works under half-wave conditions, so a phase delay of $\pi$ will be achieved. The right-handed circularly polarized and the left-handed polarized light can be represented by $J_{R}$ and $J_{L}$, respectively, in the following:

$$
\begin{aligned}
& J_{\mathrm{R}}=\frac{1}{\sqrt{2}}\left[\begin{array}{l}
1 \\
i
\end{array}\right] \\
& J_{\mathrm{L}}=\frac{1}{\sqrt{2}}\left[\begin{array}{c}
1 \\
-i
\end{array}\right]
\end{aligned}
$$

When passing through the compound lens, the polarization and phase will be changed as follows:

$$
\begin{aligned}
& {\left[\begin{array}{cc}
\cos \theta & -\sin \theta \\
\sin \theta & \cos \theta
\end{array}\right]\left[\begin{array}{cc}
1 & 0 \\
0 & e^{-i \pi}
\end{array}\right]\left[\begin{array}{cc}
\cos \theta & \sin \theta \\
-\sin \theta & \cos \theta
\end{array}\right] J_{\mathrm{R}}=J_{\mathrm{L}} e^{i 2 \theta}} \\
& {\left[\begin{array}{cc}
\cos \theta & -\sin \theta \\
\sin \theta & \cos \theta
\end{array}\right]\left[\begin{array}{cc}
1 & 0 \\
0 & e^{-i \pi}
\end{array}\right]\left[\begin{array}{cc}
\cos \theta & \sin \theta \\
-\sin \theta & \cos \theta
\end{array}\right] J_{\mathrm{L}}=J_{\mathrm{R}} e^{-i 2 \theta}}
\end{aligned}
$$

where $\theta$ is the angle between the long axis of liquid crystal and the $x$-axis. According to the existing literature, $\varphi=2 \theta$.

With phase $\varphi$, we can simulate the transmission process of light; Azimuthal angle $\theta$ is used as the aligning angle for the designed compound lenses. By the relationship between output light polarizations and the gray values that we loaded onto the SLM, a grayscale image can be generated to realize the designed output light polarization distributions for aligning LC molecule directions. The grayscale image is shown in Figure $2 b$.

For a DHFLC cell operated in the low-electric-field regime, which means under the helix unwinding electric field $(\sim 4 \mathrm{~V} / \mu \mathrm{m})$, the in-plane refractive indices induced by the electric field are dominated by the Kerr-like nonlinear terms, represented as $n_{e}(E)=n_{e}+k_{1} E^{2}$, $n_{o}(E)=n_{0}+k_{2} E^{2}$, where $n_{e}\left(n_{0}\right)$ is the zero-field refractive index of extraordinary (ordinary) 
waves, and the electrooptic coupling coefficients $k_{1}$ and $k_{2}$ depend on the smectic tilt angle $\theta$, the spontaneous ferroelectric polarization $P s$ and the dielectric susceptibility of the Goldstone mode $\chi_{E}$. The parameters can be estimated as follows: $n_{e} \approx 1.67, n_{0} \approx 1.57$, $k_{1} \approx 6.03 \times 10^{-3} \mu \mathrm{m}^{2} / \mathrm{V}^{2}, k_{2} \approx-4.12 \times 10^{-3} \mu \mathrm{m}^{2} / \mathrm{V}^{2}$ [40]. Figure 3 shows the simulation of refractive index and birefringence with the changing electric field. The birefringence can vary between 0.1 and 0.26 under an electric field of 0 to $4 \mathrm{~V} / \mu \mathrm{m}$.
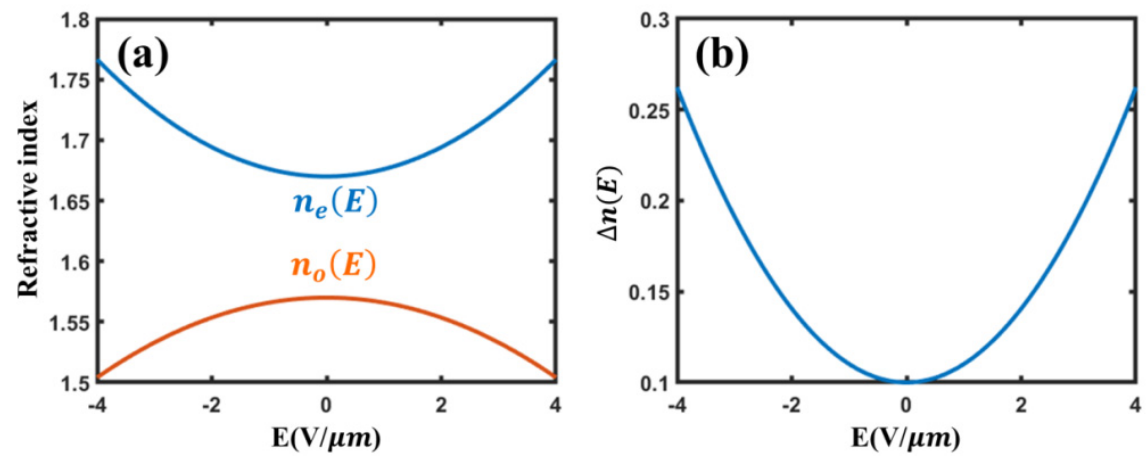

Figure 3. (a) Refractive index and (b) birefringence with applied electric field.

When left circularly polarized light passes through the compound lens, complex amplitude can be represented as follows:

$$
E=E_{1}+E_{2}=\cos \frac{\Gamma}{2} J_{\mathrm{L}}-i \sin \frac{\Gamma}{2} J_{\mathrm{R}} e^{-i 2 \theta}
$$

where $\Gamma=2 \pi \Delta n(E) d / \lambda$. Then efficiency of the compound lens can be calculated as $\eta=P_{2} /\left(P_{1}+P_{2}\right)$, where $P_{1}$ and $P_{2}$ are power of $E_{1}$ and $E_{2}$. From the simulation result in Figure 4, when cell thickness d satisfies the half-wave condition around $3.165 \mu \mathrm{m}$, the theoretical efficiency can reach its maximum of $100 \%$. However, it is not easy to fabricate this precise cell thickness as this will decrease the efficiency. As we apply an electric field of $0.75 \mathrm{~V} / \mu \mathrm{m}$, we can obtain $100 \%$ efficiency with a fast response by the DHFLC material.

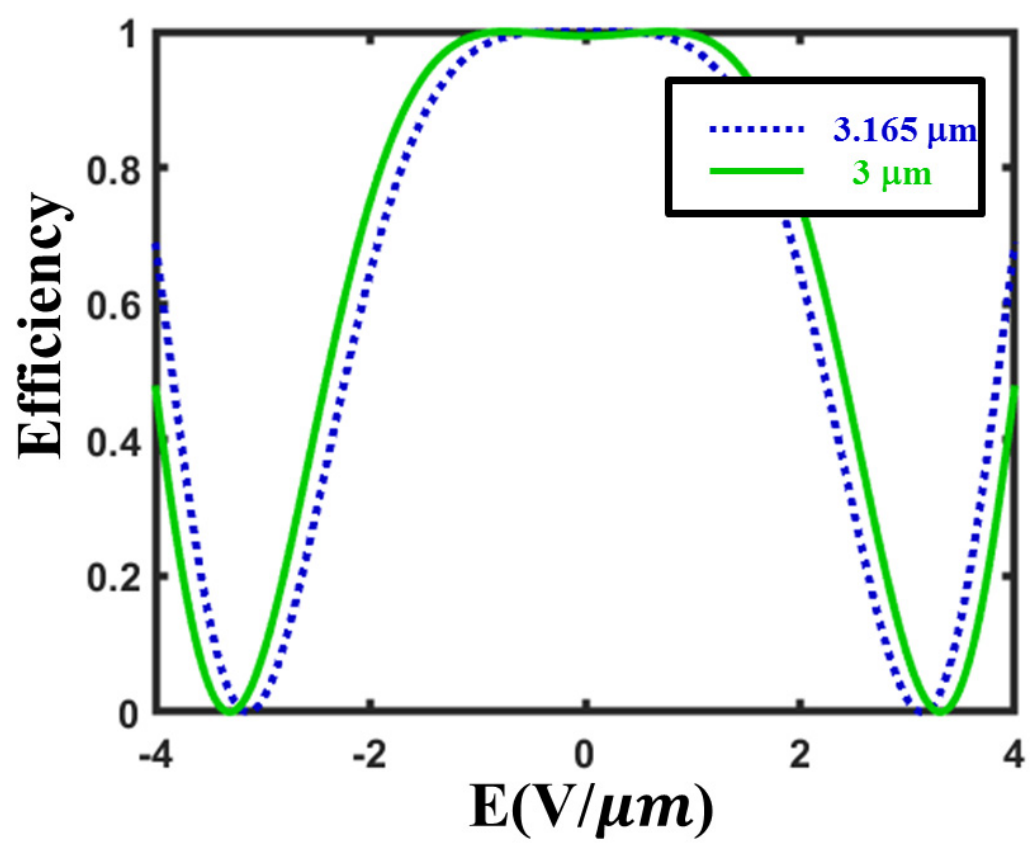

Figure 4. Simulation results of DHFLC compound lens efficiency with applied electric field. 


\section{Results and Discussion}

For DHFLC 587, the switching time can be calculated using the following equation:

$$
\tau=\frac{\gamma_{\varphi}}{K q_{0}^{2}}
$$

where rotational viscosity $\gamma_{\varphi}=0.2 \mathrm{~Pa} \cdot \mathrm{s}$ and the helix pitch $P_{0}=0.18 \mu \mathrm{m}$ at $22{ }^{\circ} \mathrm{C}$. The theoretical switching time is around $160 \mu \mathrm{s}$. In our previous work, switching time was experimentally measured at $150 \mu \mathrm{s}$ [21].

In the simulation, we set the focal length of the lens to $50 \mathrm{~cm}$ and the period of the grating to $42 \mu \mathrm{m}$. When the incident light is right-handed circularly polarized light, the output light is left-handed circularly polarized with phase $\varphi$, and the simulation results are shown in Figure 5. The light field and corresponding intensity distribution were captured at $0 \mathrm{~cm}, 10 \mathrm{~cm}, 20 \mathrm{~cm}, 30 \mathrm{~cm}, 40 \mathrm{~cm}$ and $50 \mathrm{~cm}$ behind the lens. It can be observed that as the propagation distance increases, the spot diameter increases and the light intensity decreases, showing a Gaussian distribution, so the light is divergent. At the same time, the light is diffracted to the +1 st order. When the incident light is left-handed circularly polarized light, the output light is right-handed circularly polarized with phase $-\varphi$. The simulation results are depicted in Figure 6 . We obtain the light field and intensity distribution at $0 \mathrm{~cm}, 10 \mathrm{~cm}, 20 \mathrm{~cm}, 30 \mathrm{~cm}, 40 \mathrm{~cm}, 60 \mathrm{~cm}$ and $70 \mathrm{~cm}$ behind the lens. As the propagation distance increases, the diameter of the light spot becomes smaller first and then larger, and the intensity becomes larger first and then smaller. When the distance is less than the focus length of $50 \mathrm{~cm}$, the spot diameter decreases and the intensity increases gradually. When the distance is greater than the focus length of $50 \mathrm{~cm}$, the spot diameter increases and the intensity decreases gradually. The case of the distance of $50 \mathrm{~cm}$ is not given here, because, at this position, the light field becomes an ideal point with a diameter of zero and the intensity is infinite. In this case, the light is convergent and diffracted to - 1st order. In summary, the integrated device has both the function of a lens and polarization grating.
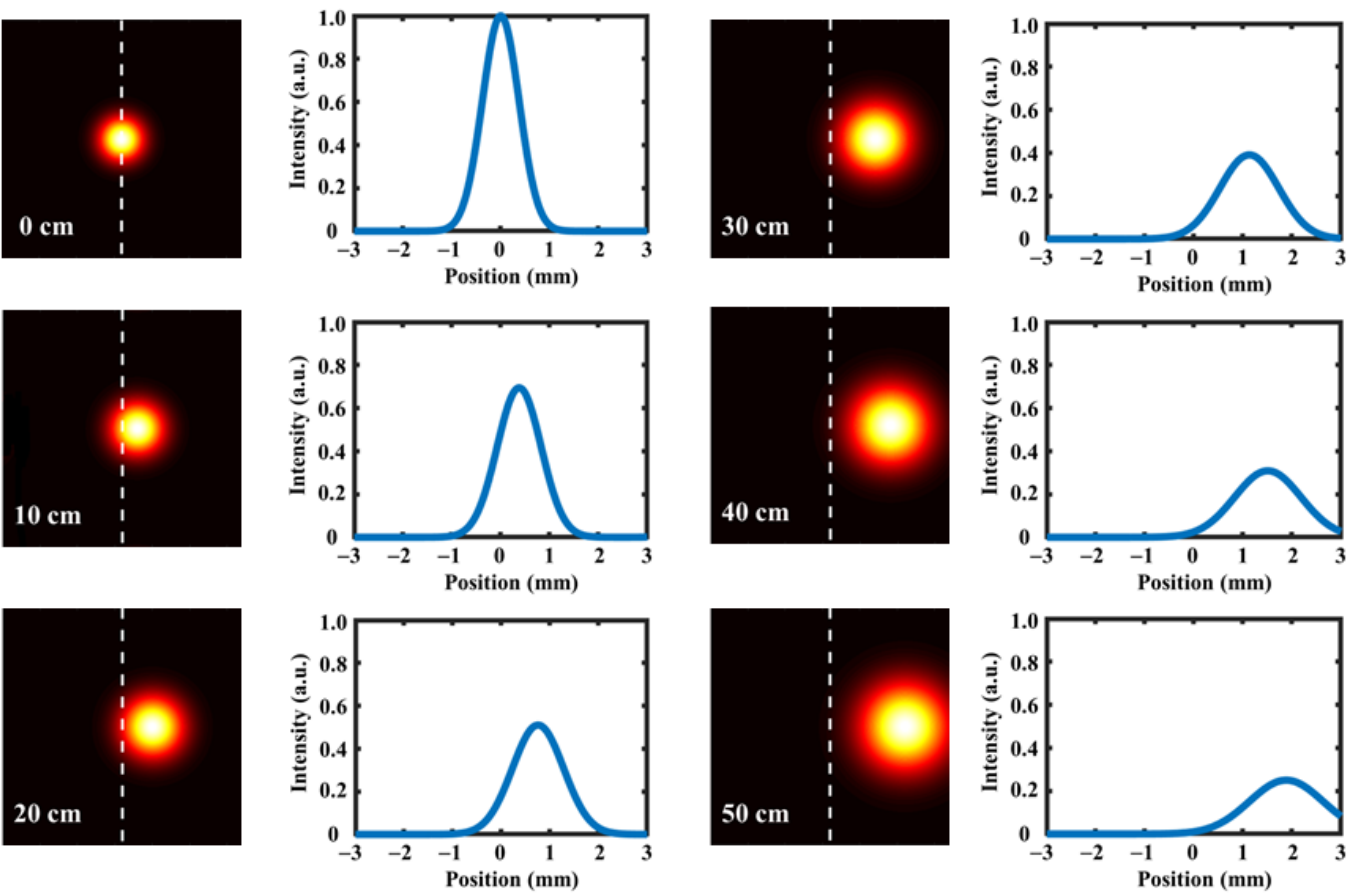

Figure 5. Simulation results of the light field and corresponding intensity distribution captured at different positions away from the compound FLC lens with right-handed circularly polarized input light. 

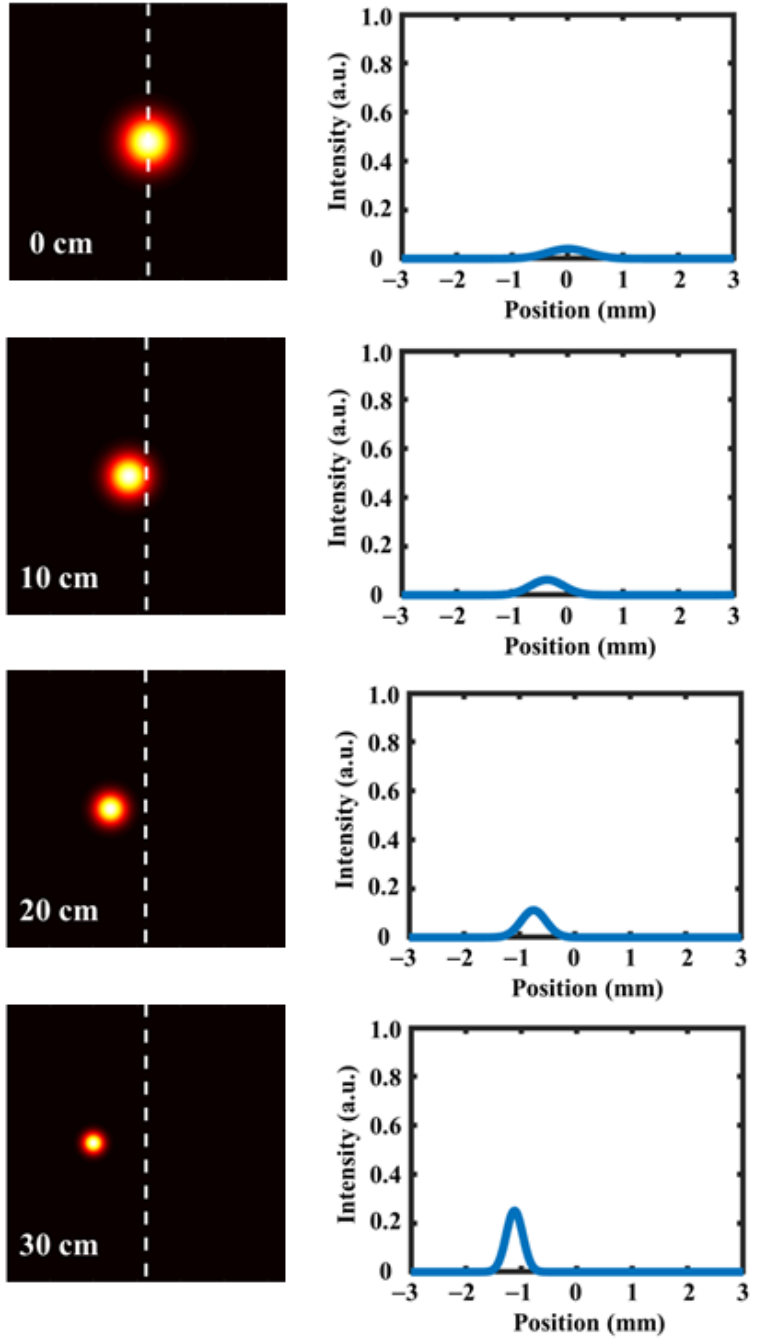

Figure 6. Simulation results of the light field and corresponding intensity distribution captured at different positions away from the compound FLC lens with left-handed circularly polarized input light.

In the experiment, we used FLC587 to fabricate the compound PB lens, and the cell gap was maintained at $3 \mu \mathrm{m}$ to meet the half-wave condition. The optical setup is illustrated in Figure 7, where an object " $\mathrm{S}$ " is placed before a QWP used to manipulate incident light polarizations. The micrograph of the fabricated lens is shown in Figure $8 \mathrm{~b}$. We tested the output light performance at the distances of $10 \mathrm{~cm}, 20 \mathrm{~cm}, 30 \mathrm{~cm}, 40 \mathrm{~cm}$ and $50 \mathrm{~cm}$ away from the lens using left-handed circularly polarized light, linearly polarized light and right-handed circularly polarized light, respectively. As shown in Figure 8a, the figures on the left side were captured under the condition of left-handed circularly polarized input light. The size of object "S" became smaller as the distance between the lens and the camera increased. The results for the right-handed circularly polarized input light are the opposite. Since linearly polarized light can be seen as a superposition of left-handed circularly polarized light and right-handed circularly polarized light, in the case of linear polarization, the output light will diverge and converge at the same time, as shown in the middle figures of Figure $8 \mathrm{a}$. We can observe that the experimental results are in good agreement with the theoretical results. 


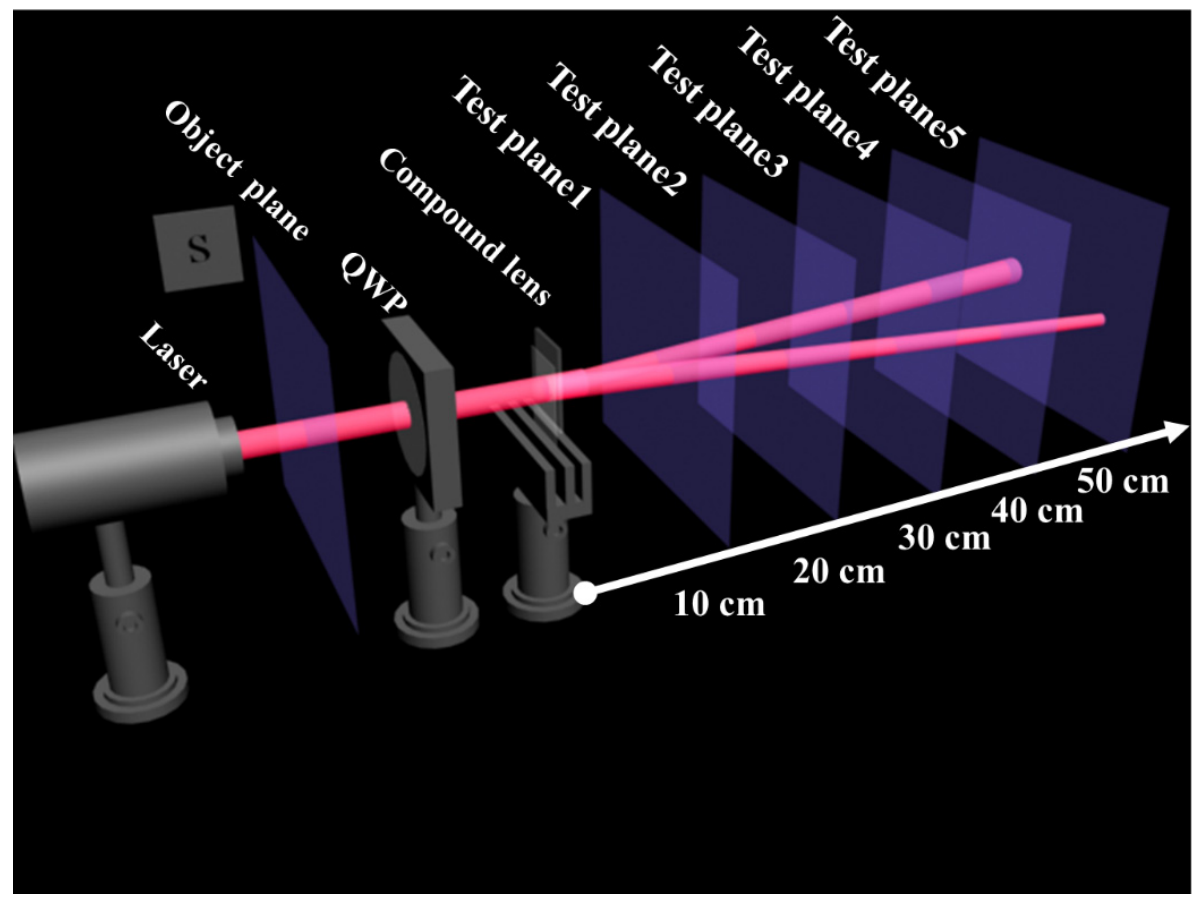

Figure 7. Illustration of experiment setups for optical performance of fabricated FLC lens.

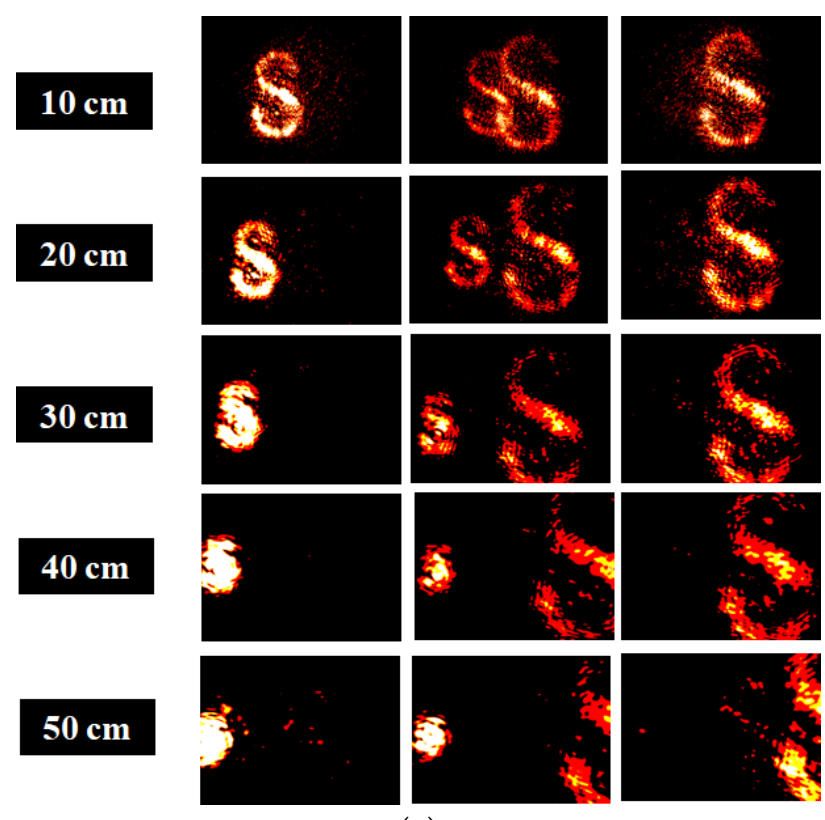

(a)

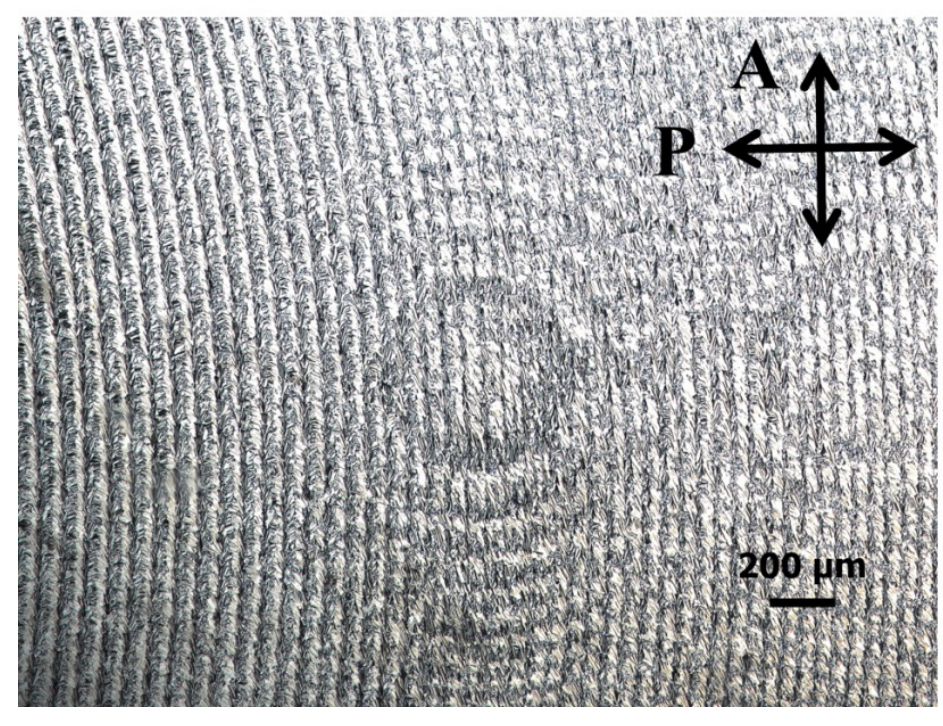

(b)

Figure 8. (a) Experimental results of the light field captured at different positions away from the FLC compound lens with left-handed circularly polarized incident light (left), linearly polarized incident light (middle) and right-handed circularly polarized incident light (right). (b) Microscopic photograph of the fabricated FLC lens.

\section{Conclusions}

In conclusion, we have demonstrated a DHFLC compound lens whose phase is an integration of a PB lens and polarization grating. We used a single-step holographic exposure setup based on an SLM as a polarization controller at the pixel level to realize continuous LC orientation. The light field and intensity distributions for different distances between the FLC lens and the camera were simulated under the input light of left-handed and right-handed circular polarizations. The experiments were conducted under the 
same conditions, with good agreement with the simulation results. Furthermore, once illuminated by a linearly polarized beam, the output light could propagate towards two directions around the central axis possessing two opposite states, namely, convergent and divergent. The DHFLC compound lenses provided a fast response time of $150 \mu \mathrm{s}$ at $2 \mathrm{~V} / \mu \mathrm{m}$. These optical features make them suitable for applications in imaging, display, beam manipulation and various optical setups that require a fast response.

Author Contributions: Conceptualization, Y.M. and M.Y.; methodology, Y.M., M.Y. and Y.S.; software, M.Y. and Y.S.; writing-review and editing, J.Z.; supervision, V.G.C., H.-S.K. and J.Z. All authors have read and agreed to the published version of the manuscript.

Funding: This research was funded by National Key R\&D Program of China, grant number 2017YFA0303800 and National Science Foundation of China (NSFC), grant number 61805202.

Institutional Review Board Statement: Not applicable.

Informed Consent Statement: Not applicable.

Data Availability Statement: All the data is contained within this article.

Conflicts of Interest: The authors declare no conflict of interest.

\section{References}

1. Naumov, A.F.; Loktev, M.Y.; Guralnik, I.R.; Vdovin, G. Liquid-crystal adaptive lenses with modal control. Opt. Lett. 1998, 23, 992-994. [CrossRef]

2. Bozinovic, N.; Yue, Y.; Ren, Y.; Tur, M.; Kristensen, P.; Huang, H.; Willner, A.E.; Ramachandran, S. Terabit-Scale Orbital Angular Momentum Mode Division Multiplexing in Fibers. Science 2013, 340, 1545-1548. [CrossRef]

3. Lei, T.; Zhang, M.; Li, Y.; Jia, P.; Liu, G.N.; Xu, X.; Li, Z.; Min, C.; Lin, J.; Yu, C.; et al. Massive individual orbital angular momentum channels for multiplexing enabled by Dammann gratings. Light. Sci. Appl. 2015, 4, e257. [CrossRef]

4. Chen, H.; Weng, Y.; Xu, D.; Tabiryan, N.V.; Wu, S.-T. Beam steering for virtual/augmented reality displays with a cycloidal diffractive waveplate. Opt. Express 2016, 24, 7287-7298. [CrossRef]

5. Zhan, T.; Lee, Y.-H.; Wu, S.-T. High-resolution additive light field near-eye display by switchable Pancharatnam-Berry phase lenses. Opt. Express 2018, 26, 4863-4872. [CrossRef]

6. Chen, H.-S.; Wang, Y.-J.; Chen, P.-J.; Lin, Y.-H. Electrically adjustable location of a projected image in augmented reality via a liquid-crystal lens. Opt. Express 2015, 23, 28154-28162. [CrossRef] [PubMed]

7. Moon, S.; Lee, C.-K.; Nam, S.-W.; Jang, C.; Lee, G.-Y.; Seo, W.; Sung, G.; Lee, H.-S.; Lee, B. Augmented reality near-eye display using Pancharatnam-Berry phase lenses. Sci. Rep. 2019, 9, 1-10. [CrossRef] [PubMed]

8. Hecht, E. Optics, 3rd ed.; Addison-Wesley: White Plains, NY, USA, 1998.

9. Chen, P.; Wei, B.-Y.; Ji, W.; Ge, S.-J.; Hu, W.; Xu, F.; Chigrinov, V.; Lu, Y.-Q. Arbitrary and reconfigurable optical vortex generation: A high-efficiency technique using director-varying liquid crystal fork gratings. Photonics Res. 2015, 3, 133. [CrossRef]

10. Li, T.; Yang, Y.; Liu, X.; Wu, Y.; Zhou, Y.; Huang, S.; Li, X.; Huang, H. Enhanced optical edge detection based on a PancharatnamBerry flat lens with a large focal length. Opt. Lett. 2020, 45, 3681. [CrossRef]

11. Jiang, M.; Guo, Y.; Yu, H.; Zhou, Z.; Turiv, T.; Lavrentovich, O.D.; Wei, Q. Low f -Number Diffraction-Limited Pancharatnam-Berry Microlenses Enabled by Plasmonic Photopatterning of Liquid Crystal Polymers. Adv. Mater. 2019, 31, e1808028. [CrossRef]

12. Kim, J.; Oh, C.; Escuti, M.J.; Hosting, L.; Serati, S. Wide-angle nonmechanical beam steering using thin liquid crystal polarization gratings. In Advanced Wavefront Control: Methods, Devices, and Applications VI; SPIE: Bellingham, WA, USA, 2008; Volume 7093, p. 709302. [CrossRef]

13. Tam, A.M.W.; Fan, F.; Du, T.; Hu, W.; Zhang, W.; Zhao, C.; Wang, X.; Ching, K.-L.; Li, G.; Luo, H.; et al. Bifocal Optical-Vortex Lens with Sorting of the Generated Nonseparable Spin-Orbital Angular-Momentum States. Phys. Rev. Appl. 2017, 7, 034010. [CrossRef]

14. Huang, B.; Wang, Q.; Jiang, G.; Yi, J.; Tang, P.; Liu, J.; Zhao, C.; Luo, H.; Wen, S. Wavelength-locked vectorial fiber laser manipulated by Pancharatnam-Berry phase. Opt. Express 2017, 25, 30-38. [CrossRef] [PubMed]

15. Jiang, M.; Yu, H.; Feng, X.; Guo, Y.; Chaganava, I.; Turiv, T.; Lavrentovich, O.D.; Wei, Q.-H. Liquid Crystal Pancharatnam-Berry Micro-Optical Elements for Laser Beam Shaping. Adv. Opt. Mater. 2018, 6, 1800961. [CrossRef]

16. Chigrinov, V.G.; Kozenkov, V.M.; Kwok, H.S. Photoalignment of Liquid Crystalline Materials: Physics and Applications; John Wiley \& Sons: Chichester, UK, 2008; Volume 17.

17. Yaroshchuk, O.; Reznikov, Y. Photoalignment of liquid crystals: Basics and current trends. J. Mater. Chem. 2011, 22, 286-300. [CrossRef]

18. Srivastava, A.K.; Wang, X.Q.; Gong, S.Q.; Shen, D.; Lu, Y.Q.; Chigrinov, V.; Kwok, H.S. Micro-patterned photo-aligned ferroelectric liquid crystal Fresnel zone lens. Opt. Lett. 2015, 40, 1643-1646. [CrossRef]

19. Huang, D.; Pozhidaev, E.; Chigrinov, V.; Cheung, H.; Ho, Y.; Kwok, H. Photo-aligned ferroelectric liquid crystal displays based on azo-dye layers. Displays 2004, 25, 21-29. [CrossRef] 
20. Srivastava, A.K.; Hu, W.; Chigrinov, V.; Kiselev, A.D.; Lu, Y.-Q. Fast switchable grating based on orthogonal photo alignments of ferroelectric liquid crystals. Appl. Phys. Lett. 2012, 101, 031112. [CrossRef]

21. Ma, Y.; Tam, A.M.W.; Gan, X.T.; Shi, L.Y.; Srivastava, A.K.; Chigrinov, V.G.; Kwok, H.S.; Zhao, J.L. Fast switching ferroelectric liquid crystal Pancharatnam-Berry lens. Opt. Express 2019, 27, 10079-10086. [CrossRef]

22. Chen, P.; Ma, L.-L.; Duan, W.; Chen, J.; Ge, S.-J.; Zhu, Z.-H.; Tang, M.-J.; Xu, R.; Gao, W.; Li, T.; et al. Digitalizing Self-Assembled Chiral Superstructures for Optical Vortex Processing. Adv. Mater. 2018, 30, 1705865. [CrossRef]

23. Chen, P.; Lu, Y.-Q.; Hu, W. Beam shaping via photopatterned liquid crystals. Liq. Cryst. 2016, 43, 2051-2061. [CrossRef]

24. Ma, Y.; Wei, B.-Y.; Shi, L.; Srivastava, A.K.; Chigrinov, V.; Kwok, H.-S.; Hu, W.; Lu, Y.Q. Fork gratings based on ferroelectric liquid crystals. Opt. Express 2016, 24, 5822-5828. [CrossRef] [PubMed]

25. Kim, J.; Li, Y.; Miskiewicz, M.N.; Oh, C.; Kudenov, M.W.; Escuti, M.J. Fabrication of ideal geometric-phase holograms with arbitrary wavefronts. Optica 2015, 2, 958-964. [CrossRef]

26. Miskiewicz, M.N.; Escuti, M.J. Direct-writing of complex liquid crystal patterns. Opt. Express 2014, 22, 12691-12706. [CrossRef] [PubMed]

27. Miskiewicz, M.N.; Escuti, M.J. Optimization of direct-write polarization gratings. Opt. Eng. 2015, 54, 25101. [CrossRef]

28. Li, Y.; Liu, Y.; Li, S.; Zhou, P.; Zhan, T.; Chen, Q.; Su, Y.; Wu, S.-T. Single-exposure fabrication of tunable Pancharatnam-Berry devices using a dye-doped liquid crystal. Opt. Express 2019, 27, 9054-9060. [CrossRef]

29. Lee, Y.-H.; Peng, F.; Wu, S.-T. Fast-response switchable lens for 3D and wearable displays. Opt. Express 2016, $24,1668-1675$. [CrossRef]

30. Pozhidaev, E.P.; Srivastava, A.K.; Kiselev, A.D.; Chigrinov, V.G.; Vashchenko, V.V.; Krivoshey, A.I.; Minchenko, M.V.; Kwok, H.-S. Enhanced orientational Kerr effect in vertically aligned deformed helix ferroelectric liquid crystals. Opt. Lett. 2014, 39, $2900-2903$. [CrossRef]

31. Pancharatnam, S. Generalized theory of interference, and its applications. Part I. Coherent pencils. Proc. Indian Acad. Sci. Sect. A 1956, 44, 247-262. [CrossRef]

32. Lee, Y.-H.; Tan, G.; Zhan, T.; Weng, Y.; Liu, G.; Gou, F.; Peng, F.; Tabiryan, N.V.; Gauza, S.; Wu, S.-T. Recent progress in Pancharatnam-Berry phase optical elements and the applications for virtual/augmented realities. Opt. Data Process. Storage 2017, 3, 79-88. [CrossRef]

33. Jacques, S.L.; Ramella-Roman, J.C.; Lee, K. Imaging superficial tissue layers using polarized light with a hand-held camera. In Third International Conference on Photonics and Imaging in Biology and Medicine; SPIE: Bellingham, WA, USA, 2003; Volume 5254, pp. 14-24. [CrossRef]

34. Soloviev, V.Y.; Zacharakis, G.; Spiliopoulos, G.; Favicchio, R.; Correia, T.; Arridge, S.R.; Ripoll, J. Tomographic imaging with polarized light. J. Opt. Soc. Am. A 2012, 29, 980-988. [CrossRef]

35. Paterson, L.; MacDonald, M.P.; Arlt, J.; Sibbett, W.; Bryant, P.E.; Dholakia, K. Controlled Rotation of Optically Trapped Microscopic Particles. Science 2001, 292, 912-914. [CrossRef] [PubMed]

36. Bimber, O.; Emmerling, A. Multifocal projection: A multiprojector technique for increasing focal depth. IEEE Trans. Vis. Comput. Graph. 2006, 12, 658-667. [CrossRef] [PubMed]

37. Guo, Q.; Brodzeli, Z.; Pozhidaev, E.P.; Fan, F.; Chigrinov, V.G.; Kwok, H.S.; Silvestri, L.; Ladouceur, F. Fast electro-optical mode in photo-aligned reflective deformed helix ferroelectric liquid crystal cells. Opt. Lett. 2012, 37, 2343-2345. [CrossRef]

38. Pozhidaev, E.P.; Kiselev, A.D.; Srivastava, A.K.; Chigrinov, V.; Kwok, H.-S.; Minchenko, M.V. Orientational Kerr effect and phase modulation of light in deformed-helix ferroelectric liquid crystals with subwavelength pitch. Phys. Rev. E 2013, 87, 052502. [CrossRef] [PubMed]

39. Ma, Y.; Yin, M.; Shan, Y.-H.; Liu, X.-Y.; Qi, S.; Chigrinov, V.G.; Kwok, H.-S.; Zhao, J. Ferroelectric liquid crystal Pancharatnam-Berry lens with a fast control of output light's polarization-handedness. Opt. Express 2021, 29, 27472. [CrossRef]

40. Kesaev, V.V.; Kiselev, A.D. Phase-only modulation of light. Opt. Lett. 2020, 45, 6703-6706. [CrossRef] 